\title{
Transatlantica
}

Revue d'études américaines. American Studies Journal

\section{Colors and Cultures / Couleurs et Cultures}

Université de Haute-Alsace (France), Université de Bâle (Suisse), et

Université de Berkeley (États-Unis) [en ligne], 13-15 avril 2021

\section{Silya Benammar et Charlaine Ostmann}

\section{(2) OpenEdition}

12 Journals

Édition électronique

URL : https://journals.openedition.org/transatlantica/16700

DOI : $10.4000 /$ transatlantica. 16700

ISSN : $1765-2766$

Éditeur

Association française d'Etudes Américaines (AFEA)

Référence électronique

Silya Benammar et Charlaine Ostmann, "Colors and Cultures / Couleurs et Cultures », Transatlantica [En ligne], 1 | 2021, mis en ligne le 25 juin 2021, consulté le 31 janvier 2023. URL : http://

journals.openedition.org/transatlantica/16700; DOI : https://doi.org/10.4000/transatlantica.16700

Ce document a été généré automatiquement le 31 janvier 2023.

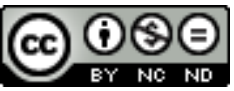

Creative Commons - Attribution - Pas d'Utilisation Commerciale - Pas de Modification 4.0 International - CC BY-NC-ND 4.0

https://creativecommons.org/licenses/by-nc-nd/4.0/ 


\title{
Colors and Cultures / Couleurs et Cultures
}

\author{
Université de Haute-Alsace (France), Université de Bâle (Suisse), et
} Université de Berkeley (États-Unis) [en ligne], 13-15 avril 2021

\author{
Silya Benammar et Charlaine Ostmann
}

1 Pendant l'événement bilingue «Colors \& Cultures - Couleurs et Cultures » qui a eu lieu du mardi 13 au jeudi 15 avril 2021, l'université de Haute-Alsace et l'université de Bâle ont accueilli virtuellement des spécialistes et non-spécialistes de la couleur, des artistes coloristes et érudits passionnés par la couleur venant de quatre continents. L'organisateur, le professeur Sämi Ludwig, assisté de sa doctorante et ingénieure pédagogique, Charlaine Ostmann, a impulsé une discussion pluridisciplinaire sur le thème de la couleur. De nombreux sujets ont été abordés, le principal étant celui de la couleur qui stigmatise les minorités en Amérique, et la couleur métaphorique utilisée comme outil de résistance en littérature et en art afro-américains, sino-américains, et natif-américains pour se libérer de l'oppression et s'accorder avec son identité crossculturelle. D'autres sujets ont émergé, tels que l'influence occidentale sur la palette et le savoir-faire traditionnels natif-américains, née des échanges commerciaux entre colons et tribus, ou encore l'influence américaine sur les cultures du monde, telles que la Chine. Pour ce compte-rendu ont été sélectionnées uniquement les interventions traitant directement de sujets en lien avec les États-Unis d'Amérique, dans l'ordre du programme.

2 Les intervenants étaient invités à s'interroger sur le rapport entre les cultures et la couleur dans un contexte américain, et ainsi à mesurer l'impact des échanges interculturels sur la couleur desdites cultures. Différentes problématiques ont été étudiées : comment la couleur est-elle nouvellement perçue dans un contexte multi et pluriculturel ? La couleur, qui est spécifique à une communauté, peut-elle être intégrée d'une culture source à une culture cible? Quel impact les migrations des cultures ontelles eu sur les couleurs traditionnelles? Aussi, comment se sont construites les couleurs traditionnelles d'une culture? Ce colloque vise à partager des perspectives 
interdisciplinaires émanant de diverses approches qui analysent les différences en perception, réception et production de la couleur.

3 La communication de Chang Liu (Université Heidelberg, Allemagne) intitulée « Who's That Girl ? On the Construction of Madonna's Star Image in Post-Mao China » porte sur la Chine post-Mao, qui a joué un rôle déterminant dans l'internationalisation de la star de la musique pop Madonna. Alors que le rouge rappelle la révolution culturelle chinoise, Madonna, la Red Singing Star ou "star chanteuse rouge", représente l'ouverture vers le monde qui suit la réforme du pays. Elle se fait connaitre en Chine dans les années 80 , lorsque la musique occidentale était définie comme pornographique ou incitatrice de comportements décadents. Elle a fait sa première apparition dans la presse chinoise dans le magazine Window of the World (1979) qui en fait un symbole d'ouverture sur le monde en Chine alors même que la presse chinoise dissimule ses apparitions osées. De même, dans le premier article majeur sorti en 1986, Madonna est avant tout présentée comme une figure héroïque socialiste antimatérialiste, issue de la classe moyenne, loin de la controverse occidentale, alors même qu'un an auparavant, la star s'affichait sur la couverture du magazine américain Playboy. L'image de Madonna est minutieusement contrôlée pour que le rouge de son titre de Red Singing Star soit associé d'une part au modèle socialiste populaire qu'elle représente, et d'autre part à sa popularité. Le "rouge Madonna» cherche donc à montrer au monde une fausse ouverture de la Chine sur l'Occident.

4 La communication "Resisting Racialized Color: U.S. Artists Challenge the Visual Regime » de la professeure Hertha D. Sweet Wong (Université de Californie, Berkeley, États-Unis) commence par une introduction complète des notions importantes en lien avec les différents aspects traités tout au long de la présentation, en particulier le terme de color-line ou «ligne de couleur». La communication de la professeur Wong étudie quatre artistes américains, la photographe afro-américaine Carrie Mae Weems, le photographe afro-américain Glenn Ligon, ainsi que l'artiste mexicain-américain James Luna, et pour finir, Nikki S. Lee, une artiste coréenne-américaine. Ces artistes dénoncent différents aspects tous plus ou moins liés au racisme, tels que l'utilisation du terme "personnes de couleur» (Colored People) dans l'œuvre éponyme de Carrie Mae Weems, dans laquelle l'artiste propose différentes photographies de personnes de couleur, en tintant chaque cliché d'une couleur monochromatique telle que le magenta, ou le jaune. Dans l'œuvre de Glenn Ligon Self-Portrait Exaggerating My White Features / Self-Portrait Exaggerating My Black Features, l'artiste nous présente deux photographies identiques qui soulignent l'absurdité des identifications raciales. Le troisième artiste abordé par Hertha Wong est James Luna, dont le travail vise à dénoncer l'oppression coloniale à l'encontre des Amérindiens, ainsi que les fausses idées qui y sont associées, tel que le Redman (littéralement l' "Homme rouge ») à travers l'humour et la satire. Nikkie S. Lee, quant à elle, dénonce les « couleurs raciales » de manière indirecte. En effet, dans une série de photographies, Lee est devenue membre de certains groupes comme celui des personnes âgées, mais aussi de la communauté hip-hop, en se créant une identité temporaire. Lee intègre une communauté en changeant sa coiffure, ou encore ses vêtements afin de devenir un membre à part entière de cette communauté. La race ou la couleur dans les œuvres de Nikkie S. Lee est donc représentée par ses expressions corporelles et ses accessoires.

5 L'intervention "Shawna Yang Ryan's Green Island: Color Imagery and the Narration of "White Terror" " de Nicoleta Alexoae-Zagni (Université Paris 8 Vincennes-Saint- 
Denis, France) se focalise sur l'autrice taïwanaise-américaine Shawna Yang Ryan et son œuvre Green Island (2016). Les éléments centraux de cette intervention sont l'analyse et la compréhension des différentes symboliques qui se cachent derrière les couleurs, ainsi que la manière dont la Terreur blanche est exposée. Cet ouvrage porte sur la période de Terreur blanche qui a eu lieu à Taïwan de 1947 à 1987. Un premier point d'analyse de Nicoleta Alexoae-Zagni est la symbolique qui entoure la couleur rouge. En effet, l'œuvre de Yang Ryan commence avec la naissance du narrateur et le sang versé lors de l'accouchement, et sa mise en relation avec le sang versé dans les rues, de par les émeutes et les combats. Dans cette première partie, le rouge est à la fois la couleur de la mort et la couleur de la vie. La prédominance de la couleur rouge peut aussi être vue comme une référence au drapeau en partie rouge de Taïwan. En effet, le rouge du drapeau symbolise le sacrifice, le sang et l'amour fraternel, qui sont des valeurs proches de celles de la République de Chine. La seconde partie de l'intervention a porté sur la couleur verte et ses différentes symboliques, en commençant par le titre de l'œuvre, qui dans un premier temps peut faire penser à la verdure abondante présente sur l'île. Néanmoins, il semblerait que le terme de Green Island soit utilisé en référence à l'île sur laquelle se trouvaient les prisonniers politiques. Ces prisons sont elles-mêmes cachées dans la verdure de l'île. La symbolique positive associée à la couleur verte est quelque peu ternie par son association avec d'autres couleurs telles que le noir ou le rouge.

6 L'intervention de Hsui-chuan Lee (National Taiwan Normal University, Taiwan) intitulée «From Racial Lines to Color Forms: The Politics of Colors in Toni Morrison's Novels " s'interroge sur la couleur noire: elle étudie les travaux de Toni Morrison, lauréate du prix Nobel, non seulement en tant qu'autrice de couleur, mais surtout en tant qu'autrice qui se soucie de la couleur. Dans le roman The Bluest Eye, l'enfant prie tous les jours afin d'obtenir des yeux bleus. Morrison oppose le bleu aux couleurs de la vie de tous les jours qui deviennent insignifiantes, alors que dans A Mercy (2008), c'est le noir qui est mis à l'écart lorsqu'une fille est choquée par la vue de la peau noire de la protagoniste, ce qui constitue pour cette dernière une première expérience du racisme pendant la période de stratification raciale dans l'Amérique du XvII siècle. God Help the Child explore la possibilité de séparer les couleurs des contraintes raciales en projetant une rencontre entre le «noir » du racisme et le «noir » de l'industrie de la mode. Alors que le racisme rabaisse la noirceur à un sens d'infériorité sociologique, la mode entretient une « liaison amoureuse » avec le " noir ». La protagoniste réapprend à voir les couleurs et à dépasser son traumatisme du noir qui la déshumanise en testant différentes combinaisons de noir et de blanc, jusqu'à ce que la barrière entre noir et blanc disparaisse. Dans le roman, une profusion de couleurs est ensuite utilisée pour décrire l'apparence physique et les vêtements des personnages post-guerre de Sécession, ce qui mène à une lecture métaphorique: les couleurs sont un moyen de rendre les noirs aussi visibles que les blancs, et ainsi de relancer la question de l'égalité des peuples face aux discours politiques post-guerre de Sécession qui avaient entièrement écarté ce sujet. Enfin, dans Home, le protagoniste Frank Money, ancien combattant coréen, subit une dépression nerveuse à son retour aux États-Unis dans les années 1950, qui le rend aveugle à toute autre couleur que le noir et le blanc. Morrison dénonce ainsi la lutte des minorités, dans ces années décrites comme pacifiques. Lee termine en expliquant qu'au-delà des œuvres de Morrison, la littérature incite le lecteur à examiner la couleur, pour la libérer des préconceptions rigides et des stratifications hiérarchisées. 
7 La communication d'Alexandra Kraeva (Université de Haute-Alsace, France) intitulée «"An Onslaught of Green / In Kodachrome 64": Middle Eastern Culture as Seen through the Prism of Color in Gjertrud Schnackenberg's "Afghan Girl” " propose une analyse du poème "Afghan Girl» de Gjertrud Schnackenberg. Elle vise à étudier les effets poétiques, mais aussi la création d'une dimension culturelle du Moyen-Orient via l'évocation de certaines couleurs évoquées par des objets. Ce poème est inspiré par la photographie mondialement connue d'une jeune fille afghane (Sharbat Gula), prise par le photographe américain Steve McCurry. Il existe deux manières principales pour désigner la couleur dans ce poème : les adjectifs liés aux couleurs, tels que "rouge » et «bleu », et les objets qui évoquent certaines couleurs, tels que le verre de mer (formé par les fragments de verre sur les plages polis par le sable), ou le camouflage. C'est ce dernier point qui fut central à la communication d'Alexandra Kraeva. En effet, plusieurs métaphores ont été relevées, telles que celle des pierres précieuses, utilisée afin de décrire les différentes nuances des yeux de Sharbat Gula. Au-delà de l'importance de l'aspect physique de Sharbat Gula, un certain nombre de métaphores semble être tourné vers les religions, avec des allusions au Nouveau Testament, mais aussi à l'Islam, religion de Sharbat Gula.

Dans sa présentation "Colour and Creole Consciousness in Jean Rhys's Wide Sargasso Sea », Alejandro Nadal Ruiz (Université de Zaragoza, Espagne) se focalise sur le roman de Jean Rhys Wide Sargasso Sea (1966) et sur la manière dont la palette de couleurs est utilisée comme outil de résistance contre la pensée coloniale réductrice. L'autrice était sensible à la couleur depuis son enfance en tant que descendante blanche de colonisateurs et membre d'une famille jadis propriétaire d'esclaves, ce qui avait accru son sentiment d'aliénation parmi ses pairs des Caraïbes. Alejandro Nadal Ruiz explore ensuite le concept d'identité fractale proposé par Cristina-Georgiana Voicu, qu'elle définit comme un hologramme composé de pièces assemblées qui forment un " tout »: l'identité d'une personne. Même si certaines pièces de l'hologramme sont détruites, les caractéristiques du «tout» de la personne sont préservées de manière identique. Le roman Wide Sargasso Sea est une réécriture de Jane Eyre d'un point de vue créole, celui de la femme créole soumise au silence par son mari Rochester, Bertha Mason, renommée Antoinette Cosway. La dualité des couleurs identitaires d'Antoinette est exprimée par une opposition entre les couleurs lumineuses, qui représentent son identité créole et sont perçues de manière positive, comme emplies de vie, et les couleurs obscures, qui désignent son identité britannique et sont perçues comme ternes, sans vie. Les couleurs deviennent pour Antoinette un moyen de se battre contre le réductionnisme de Rochester. La communication s'est conclue sur la notion d'identité fractale abordée plus tôt, transposée à Antoinette : En embrassant la couleur qui représente son identité créole, Antoinette préserve la complexité de sa crossculturalité et ainsi protège son identité, son « tout ».

Joanna Ziarkowska (Université de Warsaw, Pologne), lors de sa communication « ReClaiming Red : Eugenic Sanatoria and Strategies of Resistance in Madonna Swan: A Lakota Woman's Story » étudie l'autobiographie de Madonna Swan, racontée par Marc St Pierre. Ziarkowska commence par définir la notion de biopouvoir introduite par Michel Foucault, qui est le pouvoir que peut exercer un souverain sur ses sujets, sur leurs corps (pour les discipliner) et sur les peuples (pour les contrôler). L'autobiographie de Madonna Swan documente la politique du laissez-mourir appliquée dans les sanatoriums pendant les épidémies de tuberculoses de la fin du XIX et du début du XXe siècle, comme 
moyen de contrôler la population amérindienne. Madonna Swan raconte son calvaire lorsqu'elle contracte la tuberculose en 1944. Dans son récit, le rouge sang représente la mort de son entourage, les hémorragies subites qui précèdent la mort de ses camarades. La femme lakota intègre le Sioux Sanatorium où elle passe six années. Elle décrit y avoir subi un traitement davantage disciplinaire que thérapeutique, par l'utilisation du champ lexical de l'incarcération. Les Amérindiens ont résisté en reprenant le contrôle de leur corps, de leur mobilité, et de leur mort, en refusant les soins des sanatoriums, et en choisissant de se soigner à la maison, même si cela les mène à une mort certaine. Ils retrouvent leur identité d'Indiens lakotas, et se détachent du contrôle colonialiste oppressant.

La présentation «The Only Color the Spirits Can See: Red in Artistic Installations Memorializing Missing and Murdered Indigenous Women and Girls» de Léna RemyKovach (Université de Fribourg, Allemagne) débute par le rappel frappant que les femmes et filles indigènes sont celles qui risquent le plus de subir des violences domestiques et sexuelles aux États-Unis. Elle parcourt la couleur rouge dans les projets artistiques visant à sensibiliser le public, tels que le dispositif REDress de l'artiste métisse (Canada) Jaime Black. L'artiste suspend des robes couleur rouge sang pour rappeler que pour de nombreuses tribus d'Amérique et du Canada, le rouge est la seule couleur que les esprits peuvent voir : la couleur rouge de la robe est donc un moyen de rappeler l'esprit disparu de ces femmes et filles, afin qu'elles puissent reposer en paix. Le deuxième dispositif est appelé Walking With Our Sisters. Des centaines d'artistes et de membres de la communauté indigène ont créé ou donné des mocassins qui ont ensuite été alignés, pour dessiner un chemin. Ces mocassins jalonnent un tapis rouge et le public est invité à parcourir le chemin pieds-nus, comme pour accompagner les femmes et les filles. Le troisième projet est un partenariat entre le photographe Aaron Tambour et son modèle Michelle Buckley qui a donné naissance à une série de photographies. Elles présentent les empreintes d'une main peinte en rouge qui viennent couvrir la bouche du modèle, comme pour la faire taire. Le dernier projet artistique est celui de Maureen Gruben et son œuvre à très grande échelle, intitulée Stitching MY Landscape, qui n'est visible que depuis le ciel. Cette œuvre est composée de trous dans la banquise connectés par 3000 mètres de drap fin rouge, qui créent un motif de couture en point zigzag. Ce travail représente une alliance créative qui lie ensemble les paysages de nations disséminées à travers le continent. Ainsi, la couleur rouge symbolise l'absence des disparus car elle dénonce la justification des actes sexuels à l'encontre des femmes et filles du peuple premier.

11 Jamie K. Oxendine, administrateur de la tribu Haliwa-Saponi (Caroline du Nord), explique dans son intervention «South-East Woodland American Indian Designs \& Body Decoration: Pre-Contact and Contact » que le chiffre quatre est crucial dans les tribus. Par exemple, la roue médicinale des tribus du sud-est est composée des couleurs jaune, blanc, bleu et rouge, respectivement pour le nord, l'ouest, le sud et l'est, qui correspondent aussi aux quatre saisons, et aux quatre périodes de la vie. Il présente ensuite une illustration représentant quatre serpents à sonnette qui peuvent nager et voler dans les airs grâce à leurs ailes, orientés dans le sens du vent (sens inverse des aiguilles d'une montre) sur une roue qui représente ces directions. Dans une seconde partie, Oxendine s'attaque à certaines idées préconçues des hommes caucasiens. Il commence par présenter une illustration de deux guerriers du peuple premier dessinée par les Anglais, qui est utilisée fréquemment dans les livres d'histoires pour introduire la nation indienne pré-colonialisme. Ils sont tous deux parés du pagne, seul vêtement 
porté avant l'influence des colons, et Oxendine s'attarde sur la peau de bête avec laquelle il a été fabriqué. En effet, il affirme qu'en Caroline et en Virginie, les vêtements des tribus étaient non pas créés à partir de peau de biche comme le pense l'homme blanc, mais étaient conçus avec de la fourrure de panthère. Oxendine s'intéresse ensuite à l'art du tatouage, qui consiste à dessiner des motifs avec une arête pointue d'orphie, puis à faire pénétrer dans la peau une encre faite de charbon et d'eau ou d'huile, parfois accompagnés de pigments pour ajouter de la couleur. Parmi les nombreux motifs cités, il développe la symbolique du swastika, tatoué comme signe de bonne fortune et de prospérité. Originaire des Navajos, le symbole a voyagé vers les tribus Timucua jusqu'aux Anishinaabe, démontrant qu'il faisait partie de la culture amérindienne bien longtemps avant que l'homme blanc ne se l'approprie. Horrifiés par sa représentation raciste européenne, les tribus se sont accordées à rejeter le symbole tant qu'il demeure empreint de ces connotations négatives. L'administrateur de la tribu Haliwa-Saponi dévoile ensuite une image d'un natif enjolivé par des parures en cuivre des Grand Lacs, principal lieu où les tribus trouvaient le mica et les coquillages, avec certains endroits de la Floride et de la côte de la Caroline. Il présente les échanges et le commerce intertribaux qui permettaient de rendre accessibles des matériaux aux tribus du Sud-Ouest, ce qui a donné naissance aux routes commerciales interétatiques d'aujourd'hui, comme la route 75. Jamie Keith Oxendine termine son discours par des photographies de lui-même portant une tenue traditionnelle datant des années 1800 . Sa tenue est composée d'une longue chemise en soie, obtenue par les échanges commerciaux avec les Européens, enjolivée par des parures en argent autour du cou. Il explique qu'avant l'influence des colons, ce type de parure était fait de pierres précieuses et d'ossements, ou d'argile modelée pour en faire des perles. De même, avant que les Européens influencent la mode amérindienne, la ceinture en laine qu'il porte était faite en cuir ou en tillandsie, une plante bulbeuse aux longs épis qui étaient tissés. Sa seconde tenue, typique des guerres séminoles, présente des éléments qui ont été tissés sur les doigts (donc sans métier à tisser ou objet à tisser quelconque), comme les jarretières, la ceinture et l'écharpe qui lui servent à tenir sa corne à poudre.

12 L'artiste zuni Jaycee Nahohai (Nouveau-Mexique) propose un tour d'horizon des couleurs de son art. Le potier / céramiste et designer présente différentes œuvres et sculptures tout en expliquant son processus de création, la manière dont il a travaillé les couleurs qui proviennent de pigments naturels, quitte à acheter des matériaux (minéraux, etc.) pour ses recettes sur eBay. Le potier dévoile ses expérimentations innovantes sur la couleur. Petit-fils de la célèbre céramiste Joséphine Nahohai et fils de Randy Nahohai, qui ont remis au gout du jour les motifs et couleurs traditionnels de la tribu en poterie, Jaycee crée des œuvres qui lient tradition et modernité par un mélange harmonieux de savoir-faire ancestral, avec une maîtrise technique de la glaise, et une volonté de faire évoluer la tradition avec des expérimentations de couleurs et de matériaux colorés. Jaycee Nahohai parle notamment des teintures qu'il a ramenées de ses voyages, comme le vernis vert du Japon. Il explique les différentes techniques utilisées, comme celle du pointillisme qui donne de la texture aux plumes de ses hiboux, et fait part de la difficulté à visualiser les couleurs du produit fini car la cuisson des teintures naturelles modifie leur couleur. Il décrit ses difficultés à trouver des partenaires pour commercialiser ses œuvres depuis le début de la crise sanitaire alors que ses revenus dépendent de ses ventes, ce qui l'a même poussé à se renouveler. Il a changé provisoirement de cible et s'est ainsi spécialisé dans le design avec notamment 
la création de T-shirts stylisés aux couleurs vives qui affichent des mandalas et symboles zuni propres à la poterie, qu'il propose sur Facebook aux membres de la tribu.

13 La présentation de Sämi Ludwig (Université de Haute-Alsace, France) «Local Colors and American Multiculturalism » revisite Mumbo Jumbo de Ishmael Reed (1972) avec le postulat que le multiculturalisme du roman est associé à une multiplicité de couleurs. Ishmael Reed cherche à relocaliser l'art: dans le roman, il parle d'esthétique néovaudou, d'esthétique noire, qui est orale et perpétue une tradition et une histoire, contrairement à l'esthétique occidentale qui, avant les approches modernes, était souvent d'inspiration chrétienne. Il observe l'art noir qui est généralement animiste ou vaudou, et participe au mouvement Black Arts. Dans son roman, il peint un système polythéiste complexe, passant ainsi d'un système binaire à un système de pluralisme pour défendre une métaphysique de la multiculturalité. Ludwig compare ceci au mouvement de la couleur locale : selon Elisabeth Ammons, la couleur locale représente une allégorie littéraire de ce qui est maintenant nommé le multiculturalisme. Ludwig présente ensuite un parallèle entre le réalisme associé à la couleur locale et l'esthétique vaudou, avec de nombreux arguments. Pour en citer quelques-uns, ils sont tous deux dépourvus de théorie, associés aux concepts de minoritaire et de miniaturisme, et rattachés au terme "commun » dans les deux sens du terme, banal et non-élitiste. Le réalisme est ainsi dialogique, comme un terrain d'entente cohésif, ou une plateforme où les personnes interagissent et se connectent. Enfin, il termine avec le concept du tokenism, car la voix d'un seul auteur ne saurait représenter le point de vue de tous les Noirs : l'auteur contrebalance sa voix unique par de nombreuses illustrations qui créent une multiplicité d'expériences de vie des personnes noires. Au contraire, les représentants de la couleur locale sont seuls, et leur voix unique peut être perçue comme naïve.

14 La communication intitulée "The dissenting RED self in Lyn Hejinian's Tribunal (Omnidawn 2019), Anne Carson's Autobiography of Red (Cape Poetry,1999) \& Bhanu Kapil's Incubation: A Space for Monsters (Leon Works, 2006)» de Jennifer Kay Dick (Université de Haute-Alsace, France) porte sur la couleur rouge et sa représentation dans trois œuvres littéraires. Une première connexion est effectuée entre l'œuvre de Carson, qui débute avec un monde constitué de terre rouge, et l'œuvre de Hejinian qui commence avec une référence à l'environnement rouge de la planète Mars. De manière générale, le rouge joue un rôle central dans chacune des œuvres et reste étroitement lié à l'identité et à l'existence des différents protagonistes. Cette couleur symbolise la rage, la guerre, le désir, le sang, la honte, l'amour, et d'un point de vue politique, elle est souvent associée au parti communiste. Mais le rouge joue en plus un tout autre rôle, celui de la transformation, à l'instar de la lave, centre de la Terre, qui après son écoulement détruit et crée de nouvelles terres. Un autre point important de cette communication est la mise en évidence du lien entre écriture fictionnelle et écriture autobiographique. Durant son intervention, Jennifer Dick nous fait part des différentes interprétations possibles du rouge dans l'œuvre de Carson, le rouge renvoyant principalement au monde extérieur, contrairement à l'œuvre de Kapil, où le rouge fait constamment référence au corps.

Charlaine Ostmann (Université de Haute-Alsace, France) lors de son intervention «Colorful / Colorless: the Zuni Art of Coding Colors » s'interroge sur la couleur chez les Zuni, une tribu amérindienne localisée au Nouveau-Mexique, et sur l'impact des échanges intertribaux et avec les Euro-américains sur leurs couleurs traditionnelles. 
L'étude de trois approches, dont notamment celle de l'artiste peintre Elroy Natachu Jr., a révélé que la couleur est influencée par les théories d'artistes occidentaux tout en conservant sa tradition intra-culturelle. Ainsi, Natachu illustre sur ses toiles en acrylique son amour pour les peintres Van Gogh et Jackson Pollock, aussi présents dans ses œuvres que la palette traditionnelle zuni. De même, l'influence de ses études à l'Université du Nouveau-Mexique est visible dans les dégradés de couleurs complémentaires qui sont venus remplacer les aplats traditionnels. Au contraire, la céramiste potière Noreen Simplicio représente le noyau dur des artistes traditionalistes et se sépare de toute autre culture que celle de la tribu. Elle perpétue et conserve une tradition en répétant sur ses céramiques des motifs tels que la rosette, qui a été empruntée aux Espagnols, ou encore le deer-in-his-house. Ces symboles sont gravés dans la culture zuni depuis le xvII ${ }^{e}$ siècle par les trois couleurs qui les composent : noir et rouge sur fond blanc. Mis à part une approche plus moderne de ces symboles par un design incurvé qui remplace l'approche cubique des poteries des siècles précédents, les couleurs et formes restent figées. Enfin, l'artiste potier et DJ Jaycee Nahohai se détache par ses expérimentations, autant sur les recettes de peintures minérales et organiques naturelles que sur les teintes de ces œuvres. Ses hiboux multicolores résultent d'un travail rigoureux sur l'harmonie des couleurs naturelles et de recherche et d'échanges de matériaux autant trans-tribaux que transculturels. Sa poterie qui se démarque des normes des couleurs zuni est sa marque de fabrique, sa signature dans un marché amérindien compétitif.

Hadley Welch Jensen (Bard Graduate Center / Musée américain d'histoire naturelle, États-Unis) étudie les dye charts du peuple Diné (navajo) comme moyen d'analyser et de conserver les connaissances de la communauté lors de sa communication "Topographies of Color: Reactivating Indigenous Knowledge in Navajo Dye Charts". Les dye charts ou tableaux de teintures mettent sous verre un échantillon de tissage au centre du cadre, entouré des ingrédients (plantes et minéraux) des teintures dont les Navajos se sont servis pour colorer les fils de laine. Ces ingrédients sont séchés et collés sur le fond blanc du cadre et sont reliés au tissage par des fils, afin de dévoiler le rendu de la couleur sur la laine. Jensen commence par une contextualisation topographique des Diné, qui s'étendent du Colorado à l'Arizona et au Nouveau-Mexique. La légende raconte que le peuple a émergé des mondes enfouis sous la Terre-Mère pour atteindre Dinétah (traduisible par " parmi le peuple »), l'endroit où les peuples de la Terre et les êtres sacrés se sont rencontrés. Jensen base son étude sur les travaux de l'historienne des arts Jessica Horton, qui explique que le sens d'un paysage perceptif sculpté par des forces humaines et surhumaines est présent dans les pratiques cartographiques imaginatives des Amérindiens avant les premiers contacts avec l'occident. Jensen illustre ses propos par l'œuvre de D.Y. Begay Pollen Path (2017), avec les bandes horizontales qui rappellent les plantes et les sédiments d'un paysage du sud-ouest. Le vert sauge, le gris terreux et le dégradé de rouge sont délimités par de fines lignes couleur ocre, qui représentent le pollen de maïs. Horton soutient que ces matériaux sont des citations topographiques : à travers son tissage, Begay interprète le paysage. L'utilisation même de la laine et des teintures rappelle le paysage, les moutons de son ranch et les plantes de la région qui permettent de colorer les fils. Jensen explique que les tableaux de teintures représentent une carte mentale qui contient les connaissances historiques des endroits qui composent le paysage. Créés à l'origine dans les années 1950 par Mabel Burnside Myers (1922-1987), une tisserande navajo de Pine Springs, en Arizona, les tableaux de teinture ont permis de récolter des informations cruciales sur 
la gestion des terres et la récolte des colorants naturels utilisés par les Navajos. Un texte descriptif accompagne également chaque tableau, en anglais, parfois traduit en navajo. Ainsi, le tableau est semblable à un poster explicatif qui donne des informations supplémentaires sur les ingrédients et sur les terres de la nation navajo. Jensen souligne que bien que le contenu soit navajo, la composition du tableau révèle l'influence occidentale : les tableaux appartiennent à l'art pictural et pourraient être exposés dans les musées ou imprimés dans des livres d'histoire. Leur commercialisation et leur production de masse en font de l'art touristique ou une curiosité attractive. À travers le cadre des tableaux se trouve un microcosme des pratiques navajo visant à tisser les mondes.

17 Qingjun Li (Université de Belmont, États-Unis) analyse la couleur blanche dans le chapitre «White Tigers » du célèbre roman The Woman Warrior (1976) de Maxine Hong Kingston dans sa communication «Interpreting the Use of Color Words in Literature: A Case Study of Kingston's "White Tigers" ». Li évoque d'abord les couleurs en littérature, qui ont une fonction symbolique et émotionnelle. Dans " White Tigers », Kingston vit le fantasme de son enfance dans la peau de Fa/Hua MuLan, qui se rend dans la montagne du tigre blanc pour y apprendre les arts martiaux. Elle y forme son armée pour vaincre les officiers corrompus et rendre justice à son peuple, en volant aux riches pour donner aux pauvres. La couleur blanche surpasse toute les autres, avec la présence des animaux : la grue blanche, le tigre blanc, le lapin blanc et le cheval blanc. Li défend que le blanc est le signe d'une appropriation d'allusions déjà bien établie dans les croyances culturelles chinoises. Une des allusions mentionnées par Li est celle de la grue blanche, la raison principale du départ de Hua Mu Lan, qui agit comme un appel, une vocation. Kingston introduit le personnage de Fang Qinian, fondatrice de la Boxe de la Grue Blanche qui est adaptée aux femmes avec des mouvements proches de ceux de la grue. À travers Qinian et Hua Mu Lan, Kingston souhaite se valoriser, se rendre puissante et indépendante dans un monde dominé par les hommes. Li explore également l'allusion au Lapin blanc, qui renvoie à une légende chinoise : le lapin qui se sacrifie pour la nourrir est une métaphore. Son sacrifice la fait grandir, lui inculque des valeurs de sacrifice pour la bonne cause, et s'assure qu'elle acquière de bons traits de caractère. Ces animaux blancs accompagnent la protagoniste dans son voyage vers la maturité et vers la femme guerrière qu'elle devient, en conservant sa pureté et sa morale innocente.

La communication de Imen Yaakoubi (Université de Jendouba, Tunisie) « The Seen and the Obscene: Women, (In)Visibility, and Color From the Handmaids' Uniforms to Melania's Wardrobe" propose une analyse de l'image sociale et symbolique que renvoient les tenues vestimentaires des femmes. Elle commence par étudier la garderobe des premières dames américaines, que les médias associent à un rôle plus décoratif que politique. Ainsi, Yaakoubi affirme que le style des premières dames devient un outil pour mesurer la présidence de son époux, en citant notamment l'exemple de Jacqueline Kennedy, dont les tenues évoquent le changement générationnel, le raffinement et la haute culture. Ainsi, alors que Melania Trump prenait rarement la parole, sa garde-robe et son style ont été longuement commentés pour l'aspect superficiel et vaniteux de ses choix vestimentaires de luxe. La première dame devient ainsi une page blanche sur laquelle peuvent être commentés les échecs de la politique Trump. Yaakoubi explique que dirigeantes politiques sont soumises au même traitement médiatique, qui se focalise avant tout sur leur apparence physique, leur vie privée et leur garde-robe. Elle défend le concept de tokenism, c'est-à-dire que 
contrairement à leurs homologues masculins, les couleurs des tenues des femmes politiques sont constamment associées à une cause ou à une minorité. Ainsi, les garderobes surmédiatisées réduisent ces femmes à des gadgets pour référer à une cause, alors que la presse ignore leurs discours qui eux ne sont pas purement symboliques. Ce constat est suivi d'une étude de l'adaptation du roman The Handmaid's Tale (Margaret Atwood, 1985), en série télévisée. Alors que dans le roman les couleurs des costumes de modestie portés par les femmes de Gilead venaient de l'iconographie religieuse occidentale - les femmes en bleu pour la pureté dérivée de la couleur de la Vierge Marie, et les servantes en rouge pour le sang de l'accouchement, aussi associé à Marie Madeleine - la série défend l'idée que la couleur rouge accentue surtout le rôle des servantes qui sont principalement génitrices. La couleur devient un symbole de protestation et permet donc de rendre visible un mouvement féministe, pour protester contre le regard obscène de l'homme. Ainsi, la couleur que portent les femmes en signe de protestation donne de la visibilité en détournant les récits médiatiques et défie la surveillance politique afin de permettre aux femmes de se réapproprier leur corps.

Le colloque s'est conclu par une table ronde autour de la couleur, et par le rappel de la mise en ligne de l'intégralité des présentations sur la chaîne YouTube. La publication des articles issus des communications dans un ouvrage collectif est prévue courant 2022 / 2023. Pour plus d'information sur l'évènement, consultez le site internet du colloque, ou suivez l'actualité post-colloque sur Twitter avec le hashtag \#couleurscultures.

\section{AUTEURS}

\section{SILYA BENAMMAR}

Université de Haute-Alsace

CHARLAINE OSTMANN

Université de Haute-Alsace 